\title{
Sub-Millimeter Displacement Sensing by Passive UHF RFID Antennas
}

\author{
C. Paggi, C. Occhiuzzi, and G. Marrocco
}

\begin{abstract}
A slotted patch is transformed into a wireless passive UHF-RFID sensor of uni-dimensional displacements by introducing a mechanic-electromagnetic modulation capable to convert sub-millimeter deformations into changes of the antenna's response, remotely detectable. A design methodology allows to obtain the desired sensitivity and dynamic range in a fully controllable way. The sensor and the methodology are discussed through the help of preliminary laboratory experimentations on a concrete brick, showing the possibility to achieve resolutions better than 0.1 mm with low cost readers.
\end{abstract}

Index Terms-Crack, displacement, radiofrequency identification (RFID), sensor, smart city, structural health monitoring (SHM), UHF.

\section{INTRODUCTION}

$\mathbf{T}$ HE WIRELESS monitoring of civil infrastructures' health, as well of historical and residential buildings, is collecting growing interest in the emerging disciplines of Smart City [1] and Internet of Things [2]. A pervasive network of low-cost sensors spread over buildings and able to detect and identify damage in a structure, would enable a real-time evaluation of risky conditions thus providing a distributed set of data useful to set up a remote care of the City, prevent dangerous events and not least to manage post-disaster scenarios. Conventional sensing networks, able to monitor local behavior at critical points on the structure (cracks and expansion joints), strongly rely on visual inspection or on optical fibers, strain gauges, and displacement sensors able to transduce mechanical solicitations into visual or electrical information [3]. These sensors need a complex wiring infrastructure, continuous power source and, in case of visual inspection they also require a direct involvement of the operator to periodically collect the measured data. The complexity of this kind of sensor networks can be in principle highly simplified by the introduction of wireless sensing nodes: they may include radio interfaces to conventional sensors (typically active systems) or may instead provide a direct conversion of mechanical stimuli into an electromagnetic information, such as a drift of the resonance frequency of the device or a variation of its transmitted/received power [see [4] and [5] for a rich review on wireless structural health monitoring (WSHM)]. Examples of the recent advances

Manuscript received February 26, 2013; revised September 09, 2013; accepted November 14, 2013. Date of publication November 25, 2013; date of current version January 30, 2014.

The authors are with University of Roma Tor Vergata, 00133 Roma, Italy (e-mail: occhiuzzi@radio6ense.com; gaetano.marrocco@uniroma2.it).

Digital Object Identifier 10.1109/TAP.2013.2292520 in this area include stretchable antennas [6], [7], resonators [8], and arrays [9] eventually doped by sensitive materials such as carbon nano-structures [10]. In some cases, SHM sensors are buried into the concrete and first experiments concerning their wireless powering can be found in [11] (microwave power transmission) and in [12] (magnetic resonance coupling).

Very recently, some papers addressed the potentiality of the UHF radiofrequency identification (RFID) technology for passive WSHM purposes. The sensing capability is achieved by processing the modulated backscattering of one or more antennas (tags) equipped with microchip transponders that reply to an interrogation signal coming from a remote reader. In [13] the authors proposed a deformable meandered RFID tag (MLA) whose impedance and gain depend on the experienced deformation: if the MLA is subjected to an external longitudinal force, the antenna's shape factor changes, hence modifying the power backscattered toward the reader with a resolution down to $0.2 \mathrm{~mm}$. The dynamic range and sensitivity can be in principle adjusted by acting onto the geometrical layout of the antenna. In [14] an ink-jet printed dipole-like antenna was used to sense an applied deformation through changes in the effective conductivity of the ink that in turn produce a modulation of the backscattered power. The detuning effect of a moving metal plate behind a dipole-like tag has been instead investigated in [15] to achieve a displacement sensor. Unfortunately, dipole-like sensors are not well suited to application on concrete, metals and lossy materials which would strongly degrade the read range. A more shielded structure, proposed in [16], consists of an RFID patch antenna that relates strain to a change of resonant frequency. The device may in principle enable down to $0.1 \mathrm{~mm}$ resolution provided that the crack evolution is such to break the sensor. However, the flat profile of the antenna response makes the tag suitable to react only to high strain values. Furthermore, the frequency-shift imaging looks useful only in those Countries where the available bandwidth for UHF RFID is significant, such as USA (902-928 MHz), while its application is less effective in Europe due to the very modest allowed bandwidth (865-868 MHz). In most of the considered examples, no attempt was done to control and amplify the achieved sensitivity. A different approach is based on chip-less configurations as in [17] where a broadband antenna is provided with a delay line that can be physically interrupted by a crack thus producing a specific backscattered signature in UWB regime. The device can be considered as a two-states sensor only recognizing the presence of the crack. Finally, the possibility to monitor structural deformations by means of grids of passive RFID tags has been first investigated in [18] where 


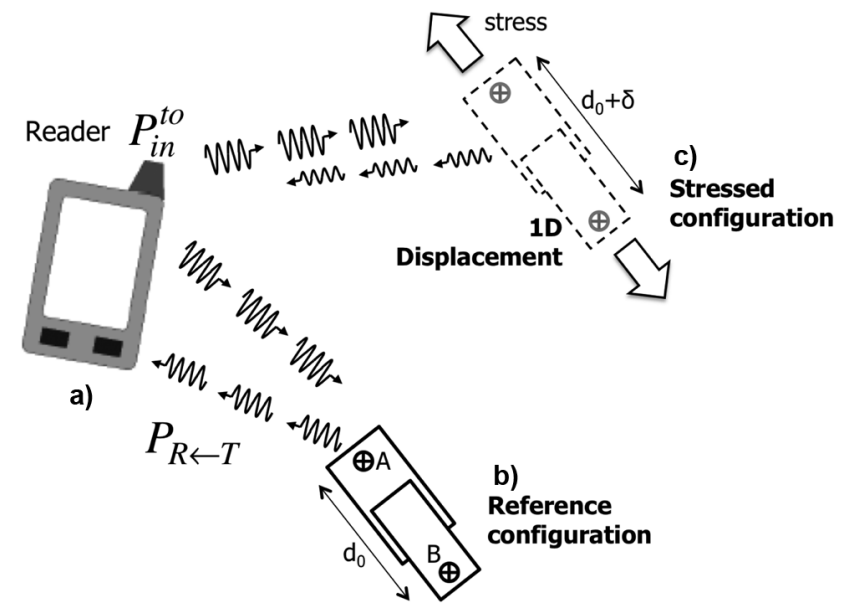

Fig. 1. a) Measurement scheme involving a reader a) for sensing of one-dimensional deformations by means of an RFID tag; b) made by two parts, independently fixed to an object at points A and B. c) It is assumed that, on application of an external force to the object, the two parts of the tag will mutually shift apart by a displacement $\delta$ with respect to the reference condition. The turn-on power and the backscattered power will be accordingly dependent on the displacement throughout the deformation of the antenna geometry.

the dependence of the tag input impedance on mutual coupling effects is engineered to sense a multi-dimensional image of surface deformation, even if with a super-millimeter resolution.

This paper introduces a very effective and fully controllable mechanism to convert small displacements of a surface into a significant change of the electromagnetic response of a UHF slotted tag (Fig. 1(a)). The key idea is to use a mechanical modulator, placed in direct contact with the radiating element, which is able to transform an induced displacement, caused by external one-dimensional forces applied to the tag along its main axis, into a change of its form factor. The mechanical modulator, depending on its shape, is also able to amplify the variation of the electromagnetic response of the antenna therefore enabling, in principle, any level of sensitivity of the whole RFID system and at least sub-millimeter resolution even with cheap readers. The description of the antenna layout comes with a design methodology to shape the response curve of the RFID radio-sensor.

This paper is organized as follows. Section II introduces the statement of the problem and the considered tag layout, whose electromagnetic response is characterized in Section III through numerical simulations. The design of the sensor, for a required sensitivity and dynamic range, is described in Section IV, and finally the sensor tag and the method itself are demonstrated in Section V by some laboratory experiments with fabricated prototypes placed over concrete.

\section{Statement of the Problem}

The design of the RFID radio-sensor is aimed at detecting the displacement among two points of an object along a linear path, due to the enlargement of a crack or to a general deformation caused by an external force. Two reference points A and B of the object (Fig. 1) are assumed to be integral with two independent parts of the antenna sensor (for instance by means of screws or by adhesive glue). The distance among these points in reference (e.g., unstressed) condition is denoted as $\overline{A B}=d_{0}$. It is more- over assumed that the application of an external force along the direction $\overrightarrow{A B}$ will produce a mutual shift of the two parts of the antenna so that the new distance among the two points becomes $\overline{A B}=d_{0}+\delta$, where $\delta$ is the one-dimensional displacement induced by the force and sensed by the antenna. The size $\overline{A B}$ can be therefore considered as a form-factor of the RFID tag, directly related to the displacement.

Sensing capability can be achieved from passive RFID tags by relating the change of the parameter under observation to the variation of some indicator obtained by processing the data measured by the reader. A detailed survey on sensing-oriented tags can be found in [19] and here, for brevity, it is only recalled that two interesting indicators are the realized gain $G_{\tau}=G \cdot \tau$ of the tag (e.g., the gain of the tag reduced by the power transfer coefficient) and the analog identifier (AID) [20], [21]

$$
\mathrm{AID}=\frac{P_{\text {chip }}}{\sqrt{P_{\text {in }}^{\text {to }} P_{R \leftarrow T}}}=\frac{2 R_{\text {chip }}}{\left|Z_{\text {chip }}+Z_{a}\right|}
$$

that is derived by processing the turn-on power $P_{\mathrm{in}}^{\text {to }}$, e.g., the minimum input power of the reader's antenna so that the power collected by the tag's microchip is enough to activate and reply back, and the power $P_{R \leftarrow T}$ backscattered from the tag to the reader. $P_{\text {chip }}$ is finally the power sensitivity of the microchip. The AID includes both direct and inverse-link data and was theoretically and experimentally demonstrated to be independent on the mutual position between the reader and the tag. The AID is simply related to the impedance $Z_{\text {chip }}$ of the microchip and to the equivalent radiofrequency impedance $Z_{a}$ of the tag.

It is worth considering that the tag response is strongly dependent on the shape of the tag itself and hence on the form factor $\overline{A B}$. The sensing metrics can be therefore related to the displacement $\delta$ by a differential measurement with respect to the initial (unstressed) condition

$$
\begin{aligned}
\Delta \mathrm{AID}[\delta] & =\frac{\operatorname{AID}\left[d_{0}+\delta\right]}{\operatorname{AID}\left[d_{0}\right]} \\
\Delta G \tau[\delta] & =\frac{G \tau\left[d_{0}+\delta\right]}{G \tau\left[d_{0}\right]} .
\end{aligned}
$$

The antenna design challenge is hence finding an electro-mechanical way to convert the displacement experienced by the structure to be monitored into a controlled variation of the realized gain and AID of an RFID tag.

\section{RFID TAG'S LAYOUT}

The proposed sensing tag is derived from a patch-like antenna since the choice of a shielded geometry provides a better immunity to the losses of typical concrete than the previously considered MLA strain-gauge [13]. Carving slots, slits and notches over a patch antenna is a common way to modify the path of surface currents and hence to control the input impedance with the purpose to miniaturize and tune a tag, as for instance in [22]. Based on the previous idea, the proposed layout includes a method to modify the shape-factor of loading notches depending on an applied displacement. More specifically, the tag (Fig. 2) is an open-circuit microstrip connected to the microchip through a slit (derived from [23]-[26]) and loaded by a pair of notches. The superior metallic plates are connected to the 


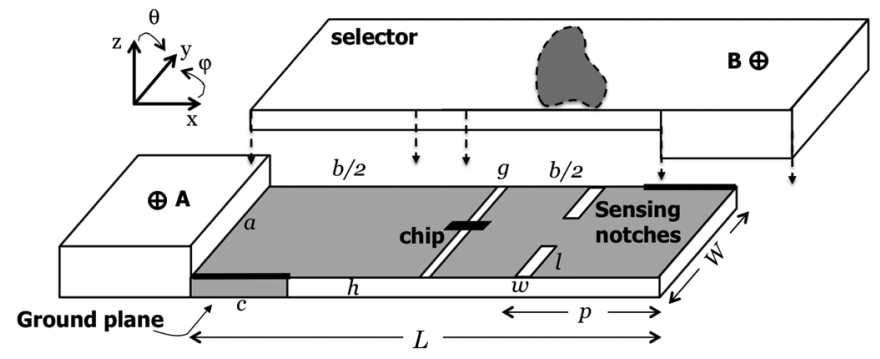

Fig. 2. Layout of the deformation-sensing tag. An open circuit slit is carved over a metallic plate connected to a ground plane by means of two vertical stripes whose length $c$ can be adjusted to achieve the required resonant frequency. The RFID microchip is placed in the middle of the slit. The upper metallization is loaded by two notches that deflect the current's path. The form factor of the two notches is modified by a metallization over a dielectric slab (the selector) placed in direct contact with the patch and free to translate with respect to it depending on the applied force. Sizes in [mm]: $a=W=32, L=78, b=77, c=18$, $g=1, h=3, l=14.5, p=19, w=1$.

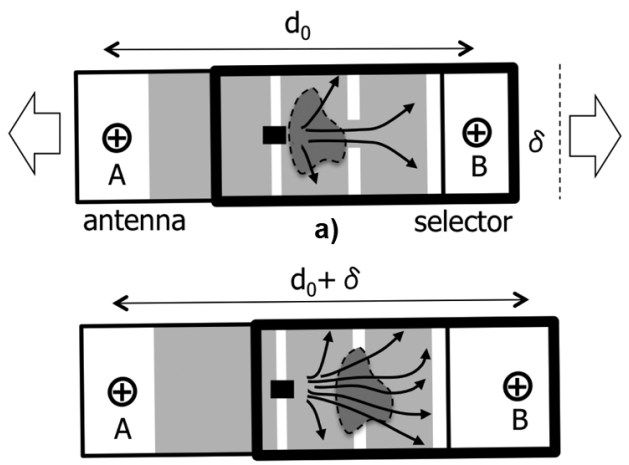

b)

Fig. 3. Pictorial representation of the change in the electric current distribution over the patch produced by a mutual shift among antenna and selector, as a consequence of an external force: a) reference condition; b) stressed condition producing a displacement $\delta$.

ground plane by two metal stripes at opposite corners, whose length can be adjusted to tune the working frequency of the antenna as in [26]. The conversion of the displacement among A, B points into a change of the notches' position $(p)$, length $(l)$, or width $(w)$ is enforced by a dielectric slab, hereafter referred to as "the selector", having the same size of the tag, suitable to be applied in contact with it and hosting a shaped planar conducting element. The tag and the selector are independently connected to the surface to be monitored at points $\mathrm{A}$ and $\mathrm{B}$ and the selector is allowed to translate parallel to the dominant size of the tag if an external force is applied. Accordingly, the relative shift of the selector will induce a continuous change of the notches' form factor (Fig. 3). A one-dimensional deformation will therefore produce a modification of the current's paths over the tag and, accordingly, the reader will detect a variation of the measured indicators AID or realized gain.

The conversion displacement $\leftrightarrow$ slot's form factor can be fully controlled by a proper design of the selector. Three possible examples, shown in Fig. 4, are suitable to modify:

a) the position $p(\delta)$ of the notches through a slitted selector;

b) the width $w(\delta)$ by using a rectangular selector;

c) the length $l(\delta)$ by a tapered (triangular) selector.

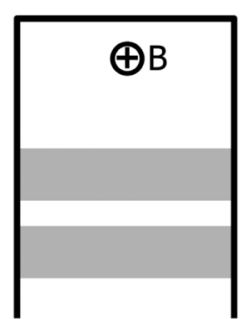

a)

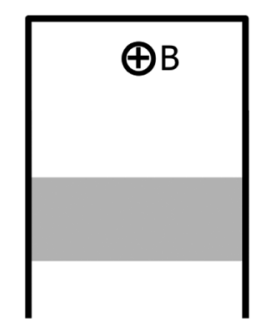

b)

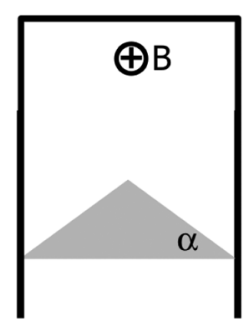

c)
Fig. 4. Kinds of selectors able to turn a one-dimensional displacement $\delta$ into a change of: a) the position $p(\delta)$-slitted selector; b) the width $w(\delta)$-rectangular selector; c) and the length $l(\delta)$-tapered selector of the notches.
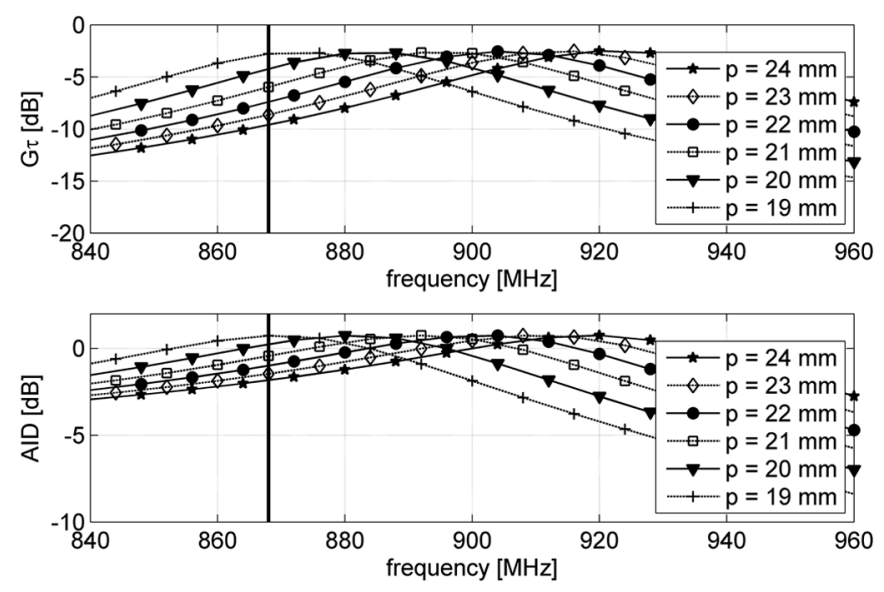

Fig. 5. Change in the notches' position $p$. (Top) realized gain and (Bottom) AID. Here $w=1 \mathrm{~mm}$ and $l=14.5 \mathrm{~mm}$.

\section{Parametric Analysis of the Sensitivity TO DISPLACEMENT}

The sensitivity of the tag response to variation of the form factor of the notches is here numerically evaluated by means of a finite-difference time-domain model simulating the effects of the three types of selectors in Fig. 4. The size of the tag in Fig. 2 has been adjusted in order to move the peak of the realized gain inside the European Band. The substrate is assumed as expanded Polyvinyl Chloride (Forex, $\epsilon_{r}=1.55, \sigma \simeq 6 \times 10^{4} \mathrm{~S} / \mathrm{m}$ ) and the reference impedance of RFID microchip is $Z_{\text {chip }}=13+$ $j 151 \Omega$ (Inpinj Monza 4QT, having power sensitivity $P_{\text {chip }}=$ $-18 \mathrm{dBm}$ ). In all the considered simulations, the tag is placed over a dielectric box representing the mature concrete $\left(\epsilon_{r}=4.9\right.$, $\sigma \simeq 5 \times 10^{-3} \mathrm{~S} / \mathrm{m}$ ) [27].

Figs. 5-7 show the variation of the antenna response when each parameter $p, w, l$ of the two notches has been gradually changed with respect to the reference condition $\{w(0)=5 \mathrm{~mm}$, $l(0)=14.5 \mathrm{~mm}, p(0)=19 \mathrm{~mm}\}$. It is worth noticing that the dependence of the realized gain and of AID on the geometrical variations are monotonic. In the European Band in particular, as the notches move away from the middle of the tag ( $p=$ $19 \rightarrow 25 \mathrm{~mm}$ ) the performance indicators decrease as well as if the notches become shorter $(l=14.5 \rightarrow 0 \mathrm{~mm})$ or wider $(w=1 \rightarrow 5 \mathrm{~mm})$. Opposite trends are visible in the FCC band (902-928 MHz). 

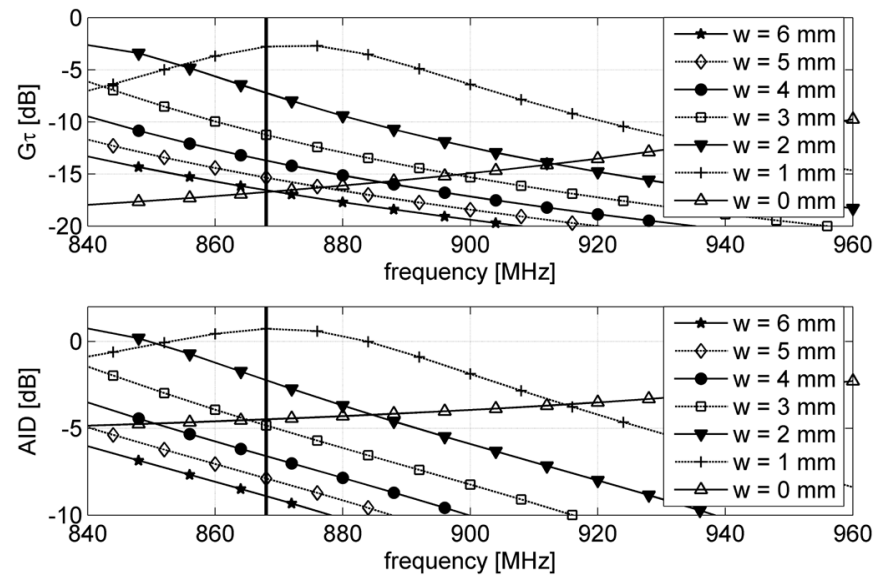

Fig. 6. Change in the notches' width $w$. (Top) realized gain and (Bottom) AID. Here $p=19 \mathrm{~mm}$ and $l=14.5 \mathrm{~mm}$.
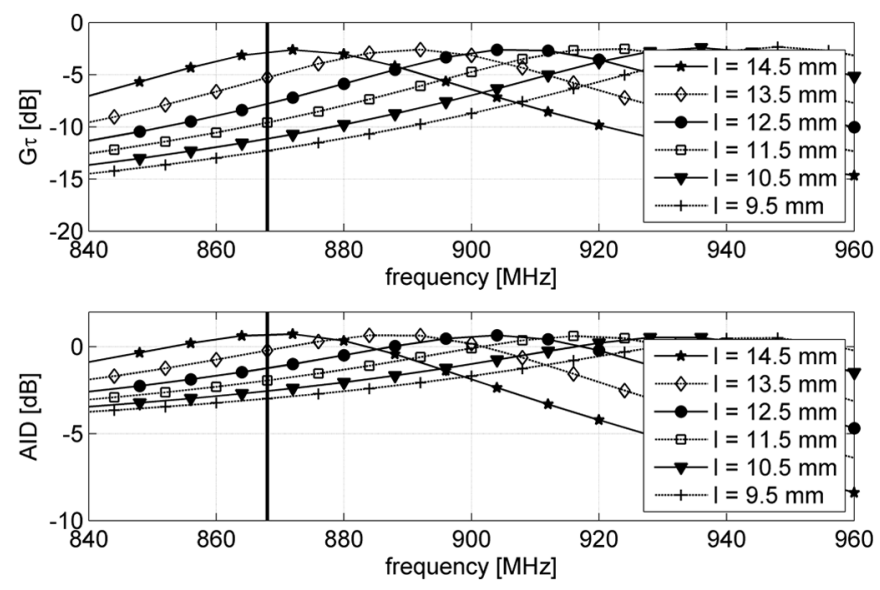

Fig. 7. Change in the slot's length $l$. (Top) realized gain and (Bottom) AID. Here $p=19 \mathrm{~mm}$ and $w=1 \mathrm{~mm}$.

Fig. 8 finally summarizes the gain and AID response evaluated at $868 \mathrm{MHz}$ versus overall $5 \mathrm{~mm}$ variation of notches parameters referred to the initial condition $\{w(0), l(0), p(0)\}$ defined above. It is apparent that the conversion of physical deformations into a change of the notches' width $(\Delta w)$ or length $(\Delta l)$ will provide the most effective change of the antenna response. It is moreover worth noticing from Fig. 6 that the change of the realized gain in the two configurations $w=1 \mathrm{~mm}=>w=0$ (no notches) is more than $10 \mathrm{~dB}$, e.g., large enough to potentially forbid the communication with the reader in the $w=0$ case. Such a property can be engineered to achieve a one-bit deformation sensor, able to transmit its ID to the reader only when the experienced deformation exceeds a given threshold easily programmable through the width of the notches and by using the rectangular selector in Fig. 4(b).

\section{Design Methodology}

The design of the RFID-sensor having a given sensitivity and dynamic range of the deformation under observation can be reduced to a proper shaping of the selector. Among the three considered layouts in Fig. 4, the tapered one (case c) is able to convert a displacement along $x$-direction (see Fig. 3 ) into an action occurring along $y$-direction whose entity depends on the
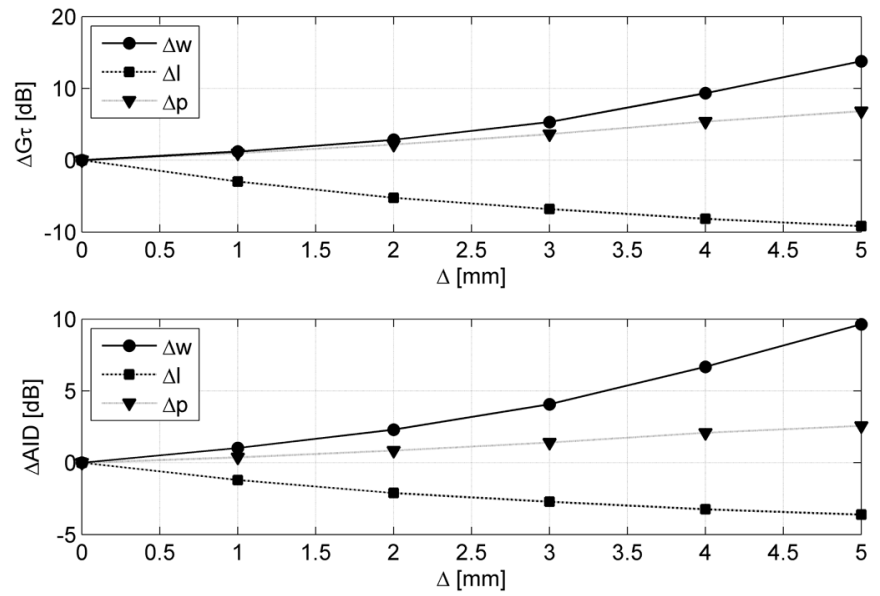

Fig. 8. Change of the tag's electromagnetic parameters at $868 \mathrm{MHz}$ for overall $5 \mathrm{~mm}$ variation of the parameters of the sensing slots.

tapering angle of the selector. The parameters of the selector can be hence engineered with the purpose to amplify the effect of the external displacement and match a required sensitivity and dynamic range of the RFID sensor.

A possible design procedure, suitable to any tag layout that includes tuning notches, is here described ${ }^{\prime \prime}$, provided that a diagram/table (as in Fig. 8) of the relationship $\left\{\Delta\right.$ AID, $\left.\Delta G_{\tau}\right\} \leftrightarrow l$ has been made available by simulations or/and measurements. The method is exposed for the case of linear tapered selector as in Fig. 4(c), but it can be simply extended to more complex profiles as shown later on.

\section{A. Linear Tapered Selector}

Let $\delta_{1}$ denote the maximum displacement that is requested to be observed through sensitivity $S[\mathrm{~dB} / \mathrm{mm}]$ in compliance with the resolution of the specific RFID reader and with the power budget (see [28] and [29] for a detailed discussion on this issue). Accordingly, the RFID reader has to appreciate a minimum dynamic range

$$
\Delta \xi_{\min }=S \delta_{1}
$$

of the electromagnetic indicator $\xi=\{G \tau$, AID $\}$.

If $\left[l, l_{0}\right]$ is the variation of the length of the antenna's notches required to produce the dynamic range $\Delta \xi_{m i n}$, then the tapering angle $\alpha$ of the triangular selector is such to map the whole span of the RFID signal $\Delta \xi_{\min }$ into the interesting range of displacement. From the geometrical construction of Fig. 9 the following equation holds:

$$
\tan \alpha=\frac{\delta_{1}}{\Delta l}
$$

with $\Delta l=l_{0}-l_{1}$. A roughly linear relationship $\left.\Delta \xi_{\min }\right|_{\mathrm{dB}}=$ $K \Delta l$ is assumed, so that $K$ is the sensitivity of the device to the variation of the notches' length as derived by Fig. 8 and is a feature of the specific antenna layout. The tapered selector will therefore produce an amplification $S=K \tan \alpha$ fully controlled by the selector's angle

$$
\alpha=a \tan \frac{K}{S} .
$$




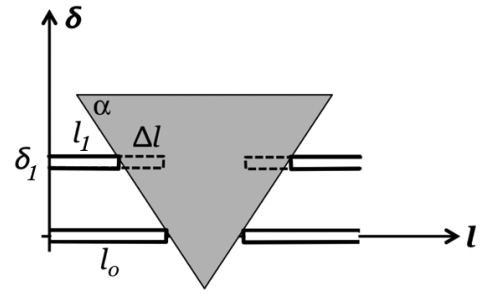

Fig. 9. Geometrical relationship to derive the tapering angle $\alpha$ of the tapered selector in order to map all the required variation of electromagnetic indicator $\Delta \xi_{\text {min }}$, when the notches's size change as $l_{1} \leq l \leq l_{0}$, to the maximum deformation $\delta_{1}$ to be sensed.

Since $\tan \alpha$ may achieve any value, the device is theoretically able to provide an as small as desired sensitivity. Having fixed sensing requirements $\left\{\delta_{1}, S\right\}$, the design of the RFID sensor can be therefore performed according to the following three steps:

i) design the slitted patch to work at the RFID frequency in the reference condition $\{w(\delta=0), l(0), p(0)\}$ and identify the maximum and minimum lengths of the sensing notches such to achieve a dynamic range of the antenna response (realized gain or AID) not less than $\Delta \xi_{\text {min }}=$ $\delta_{1} S$

ii) the factor $K=\Delta \xi_{\min } / \Delta l$ is estimated by means of simulations or measurements, as described in the next example section;

iii) design the selector's tapering angle according to (6).

\section{B. Limitations to Largest Detectable Displacements}

Above formulas permit to understand the limitation to the maximum displacement that can be sensed by the proposed device, and its relationship with the requested sensitivity.

Equation (5) indicates that the maximum displacement can be theoretically as large as desired by letting the angle $\alpha \rightarrow \pi / 2$, e.g., by means of a very sharp selector. However, the geometrical compatibility of the selector with the radiating part of the tag (see Figs. 2 and 9) will request that $\delta_{1}<(b / 2-p)$ in order to the microchip not to be short-circuited by the selector itself. Moreover, (4) trivially tells that sensitivity and maximum displacement are opposite requirements and their product is limited by the smallest signals $(\Delta \xi)$ detectable by the reader for a given distance from tag (see [28] for a comprehensive discussion about this topic). Therefore, having fixed the required sensitivity and the maximum acceptable variation of the reader's response, the maximum detectable displacement $\delta_{1}$ will be accordingly limited by (4) and can be physically implemented by properly designing the position of the sensing notches over the tag's antenna.

\section{Extension to Shaped Tapering Profiles}

More complex tapering profiles could be useful for additional freedom in the shaping of the calibration curve of the sensor. At this purpose the linear-taper selector in Fig. 9 is replaced by a piecewise-linear profile (see Fig. 10) such to map different intervals of displacement $\left\{\delta_{n}\right\}$ to specific measurement sensitivities $\left\{S_{n}\right\}$, so that displacements $\delta_{n-1} \leq \delta<\delta_{n}$ will be detected with sensitivity $S_{n}$. The selector profile will be accord-

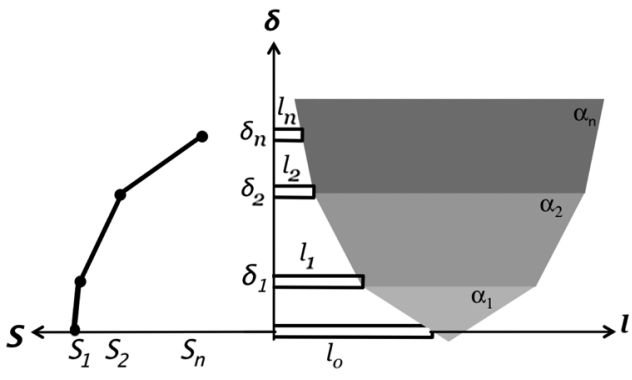

Fig. 10. Geometrical relationship to design non-uniform tapered selectors with a piecewise-linear profile capable to implement more general displacementssensitivity rules.

ingly described through angles $\left\{\alpha_{n}\right\}$. The overall design procedure is therefore arranged as a sequence of designs of linear selectors. For each $n$th segment of the selector's profile the design procedure is the following: $i$ ) identify the lengths $\left\{l_{n-1}, l_{n}\right\}$ of the notches such to achieve the dynamic range of the antenna response not less than $\left.\Delta \xi_{n}=S_{n}\left(\delta_{n}-\delta_{n-1}\right) ; i i\right)$ estimate the factor $K_{n}=\Delta \xi_{n} /\left(l_{n-1}-l_{n}\right)$; iii) calculate the angle $\alpha_{n}=a \tan \left(K_{n} / S_{n}\right)$. The procedure can be iterated for each section of the selector, hence building the whole profile. A detailed example will be presented in the next experimental section.

\section{EXPERIMENTATIONS}

The proposed layout and the design method are now evaluated by laboratory experimentations over a sensor prototype fabricated by carving an adhesive copper sheet with a digital controlled cutter, according to the sizes in Fig. 2. The whole conducting structure of the patch is suited to be foldable and hence can be obtained almost as a single part, to be folded around the substrate (Fig. 11(a)) to be folded around the substrate. Fig. 11(b)-(d) show the fabricated tag together with the selector made by plexiglass to visually check the alignment of the copper triangle during the experiments.

\section{A. Measurement Set-up}

The tag is placed over a mature concrete brick and the measurement set-up (Fig. 12) comprises a ThingMagic M5e reader connected to a linear polarized broadband PIFA antenna having $5.5 \mathrm{~dB}$ maximum gain and placed in front of the concrete brick. The ground plane has been extended to improve the electromagnetic decoupling from the medium wherein the tag is attached on.

The realized gain and Analog Identifier of the tag measured in the reference condition, e.g., in absence of displacement $(w(\delta=$ $0)=1 \mathrm{~mm}, l(0)=14.5 \mathrm{~mm}$, and $p(0)=19 \mathrm{~mm}$ ) are shown in Fig. 13, together with the simulated data. The realized gain at the European frequency is $\left.G_{\tau}(f=868 \mathrm{MHz})\right)=-2.5 \mathrm{~dB}$ corresponding to a maximum read distance of about $7 \mathrm{~m}$ in case of $3.2 \mathrm{~W}$ EIRP emitted by the reader, $-3 \mathrm{~dB}$ polarization matching and $P_{\text {chip }}=-18 \mathrm{dBm}$. The realized gain has been measured according to the turn-on method [30]. The AID has been calculated by means of the (1) for different reader-tag positions, as in [20], [21], starting from turn-on power $P_{\text {in }}^{\text {to }}$ and from the corresponding backscattered power $P_{R \leftarrow T}$ collected 


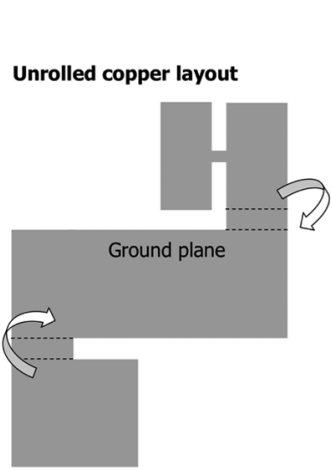

a)

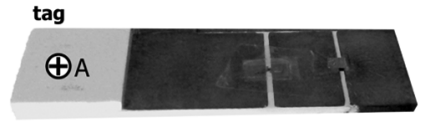

b)

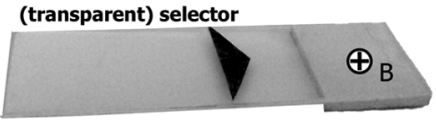

c)

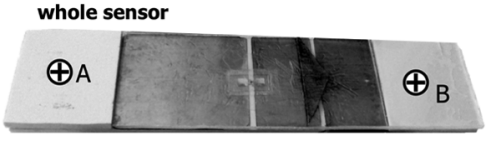

d)
Fig. 11. a) Unrolled copper layout of the prototype; b) fabricated tag; c) tapered selector made by plexiglass; and d) assembled sensor. a)

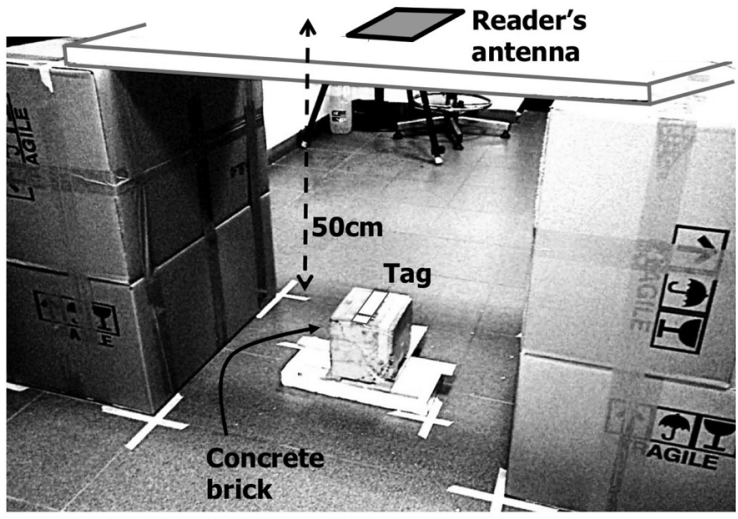

b)

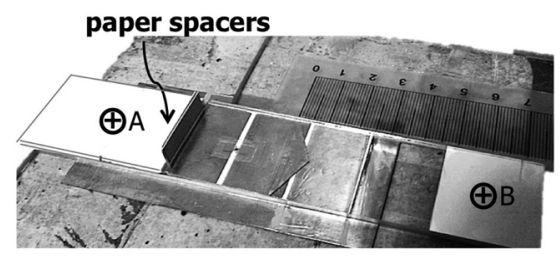

Fig. 12. a) Measurement set-up. b) Details of the sensor: external displacement is emulated by introducing $0.2 \mathrm{~mm}$ paper-sheet spacers between the RFID tag and the selector.

by the reader. In particular, the backscattered power is estimated from the received signal strength indication (RSSI) according to a reader-specific empirical conversion.

Measurements and simulations are in nice agreement in European band, while some discrepancies are visible for higher and lower frequencies because of the change of the chip impedance that is not accounted in the numerical models. The measured AID has been in particular estimated for different mutual positions of the reader-tag (angle $\theta$ and distance $r$ as in the reference system in Fig. 2) and the obtained results confirm the high level of immunity for the AID from the set-up conditions.

\section{B. Static Analysis and Characterization of the Sensing Mechanism}

To preliminarily verify the basic sensing mechanism, the simulated diagram in Fig. 8 has been reproduced by manual variation of the notches's length $l=l_{0}+\Delta l(\Delta l=0 \div 5 \mathrm{~mm})$ with respect to the initial condition by partially closing the notches

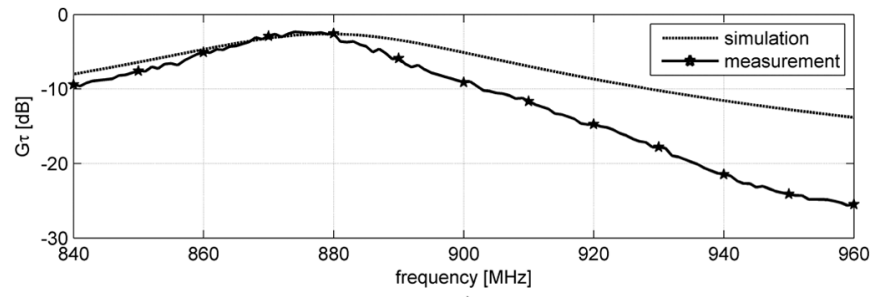

a)

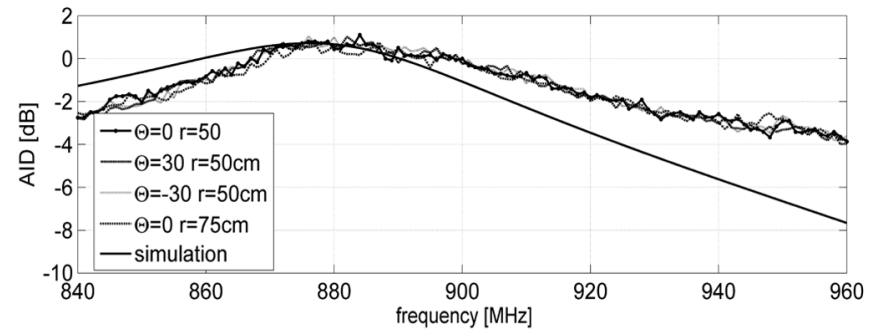

b)

Fig. 13. Simulated and measured performance of the RFID tag at $870 \mathrm{MHz}$ in the reference condition (slot's size $w(0)=1 \mathrm{~mm}, l(0)=14.5 \mathrm{~mm}$, and $p(0)=19 \mathrm{~mm}$ ). a) realized gain versus frequency at broadside observation $(\theta=0, \phi=0)$ and $b)$ Analog identifier (AID) for different measurement positions (distances: 50 and $70 \mathrm{~cm}, \theta=0^{\circ}, \pm 30^{\circ}$ ).

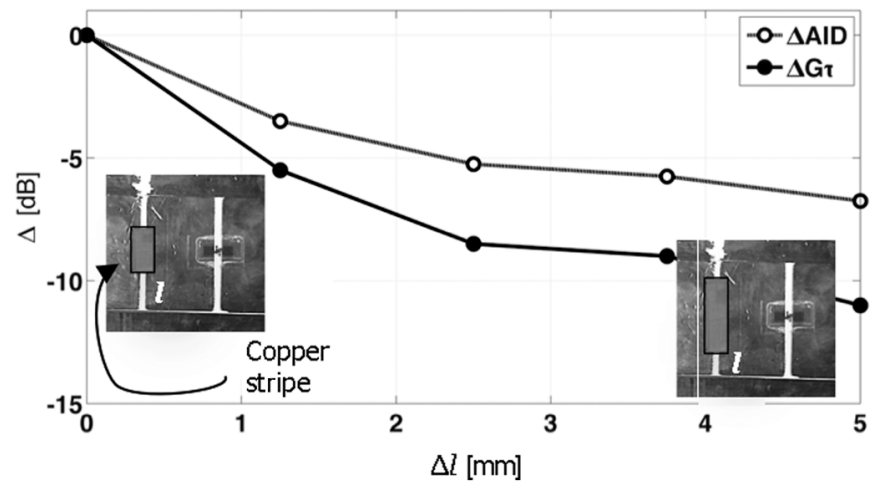

Fig. 14. Measured change of realized gain and AID for the fabricated prototype, without selector, with respect to manual variation of notches' length $l=l_{0}+\Delta l$ by adding copper stripe of increasing length.

through copper stripes of increasing length without the use of a true selector. The measured sensing profiles in Fig. 8 are in good agreement with simulations, even though the prototype appears slightly more sensitive.

\section{Design of a $4 \mathrm{~dB} / \mathrm{mm}$ Linear Selector for $1 \mathrm{~mm}$ Displacement}

The design procedure for the linear selector described in the previous Section is now applied to achieve a highly sensitive sensor with $S_{\mathrm{AID}}=4 \mathrm{~dB} / \mathrm{mm}$ over a small displacement $0<$ $\delta \leq 1 \mathrm{~mm}$. The minimum required dynamic range (4) is accordingly $\Delta \mathrm{AID}_{\min }=4 \mathrm{~dB}$. With reference to the measured calibration curve in Fig. 14, the required change of the notches' length producing $\Delta \mathrm{AID}_{\min }$ is $\Delta l \simeq 2 \mathrm{~mm}$, and accordingly, the scaling factor is $K=\Delta \mathrm{AID}_{\min } / \Delta l=2 \mathrm{~dB} / \mathrm{mm}$. The angle $\alpha$ of the tapered selector is finally estimated from (6) as $\alpha=a \tan (2 / 4)=27^{\circ}$.

In the dynamic measurements, the evolving displacement experienced by the tag has been emulated by introducing portions 

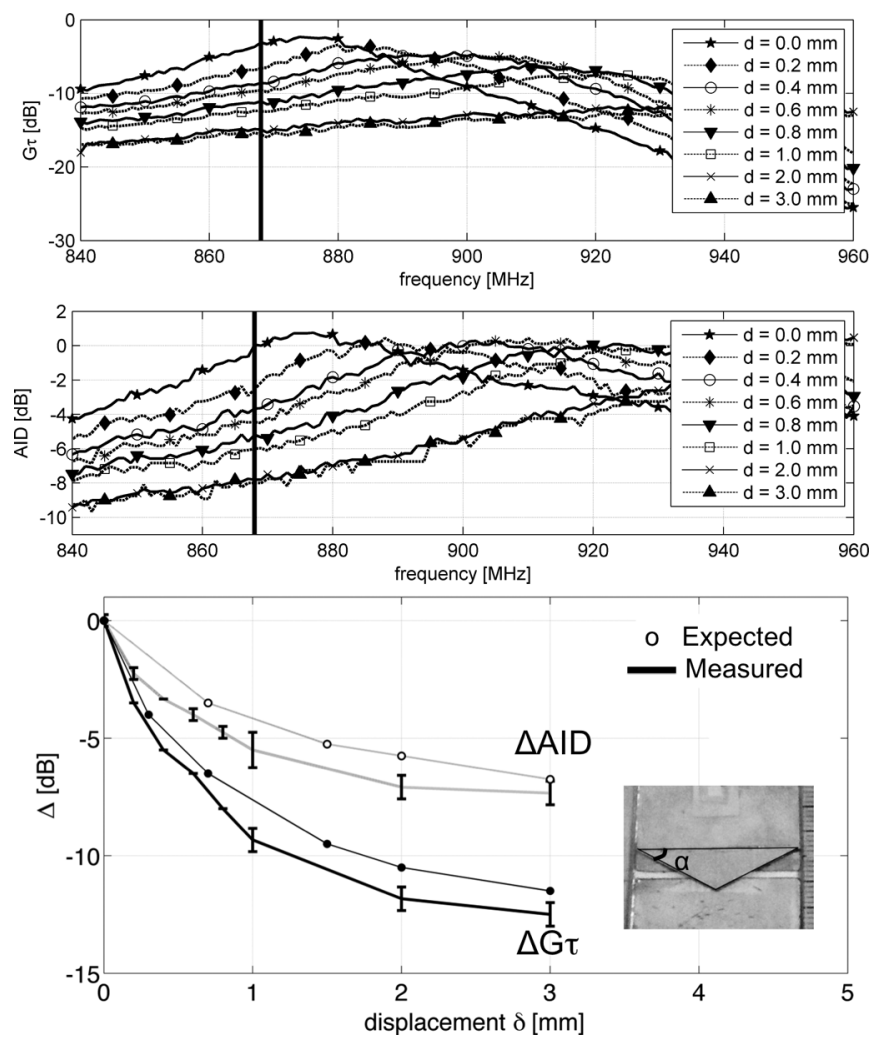

Fig. 15. Measured multi-frequency realized gain and AID versus deformation and corresponding calibration curves at $870 \mathrm{MHz}$ for the $\alpha=27^{\circ}$ selector (expected results and measurement mean and standard deviation).

of paper spacers (thickness: $0.2 \mathrm{~mm}$ ) between the tag and selector (see inset of Fig. 12) such to produce a stepped displacement $0 \leq \delta \leq 3 \mathrm{~mm}$.

Multifrequency measurements (Fig. 15) of the realized gain and AID exhibit the same type of profiles found in simulations (Fig. 7). In particular, the responses at $870 \mathrm{MHz}$ show variations $\Delta G \tau=9 \mathrm{~dB} / \mathrm{mm}$ and $\Delta \mathrm{AID}=5 \mathrm{~dB} / \mathrm{mm}$ for $0 \leq \delta \leq 1 \mathrm{~mm}$, that are in reasonable agreement with the expected profiles (as obtained by the mathematical $\delta \leftrightarrow l$ remapping). The overall variations finally tends to saturate to $12 \mathrm{~dB}$ (realized gain) and $8 \mathrm{~dB}$ (AID) in case $\delta>2 \mathrm{~mm}$, anyway outside the controlled range.

\section{Design of a Dual-Tapered Selector for $5 \mathrm{~mm}$ Displacement}

The selector is now redesigned in order to achieve a lower but constant sensitivity $S_{\mathrm{AID}}=1 \mathrm{~dB} / \mathrm{mm}$ within a wider range of displacement $0<\delta \leq 5 \mathrm{~mm}$. Diagram in Fig. 14 shows an intrinsic saturation of the device response versus the change of the notches' length and therefore a triangular selector, that produces a linear remapping of the calibration curve $\Delta \xi \leftrightarrow$ $\Delta l \leftrightarrow \delta$, is not adequate to the scope. A dual-tapered selector (in the sense of Fig. 10) is hence considered in order to equalize the response of the RFID sensor, e.g., to attenuate the sensitivity to small displacements and to amplify that to larger displacements.

The selector will be characterized by two angles $\left\{\alpha_{1}, \alpha_{2}\right\}$ to be determined according to the procedure in Section V-C with the requirements that $S_{1,2}=\{1,1\} \mathrm{dB} / \mathrm{mm}$ and

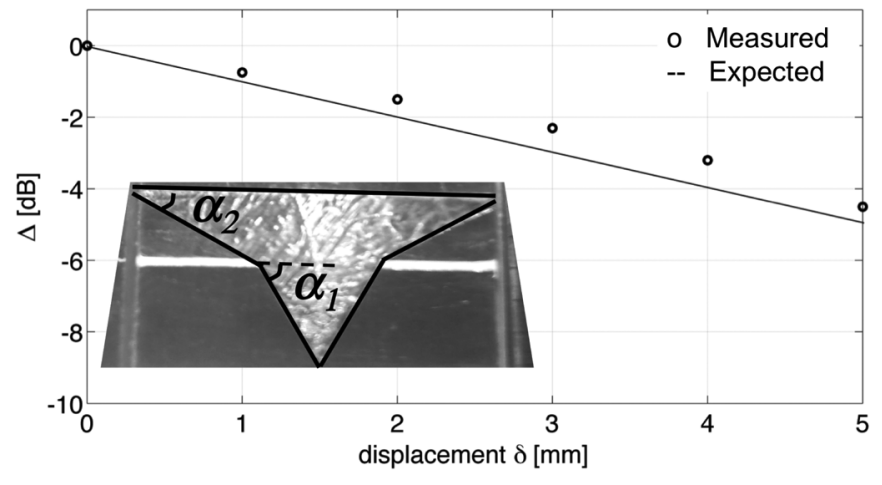

Fig. 16. Measured calibration curves $\triangle \mathrm{AID} \leftrightarrow \delta$ at $870 \mathrm{MHz}$ for the dual-tapered selector with angles $\alpha_{1}=70^{\circ}$ and $\alpha_{2}=45^{\circ}$. The straight line indicates the desired $1 \mathrm{~dB} / \mathrm{mm}$ sensitivity.

$\delta_{1.2}=\{2,5\} \mathrm{mm}$. By application of (4) the minimum required dynamic ranges are $\Delta \mathrm{AID}_{1,2, \min }=\{2,3\} \mathrm{dB}$ and, from the measured calibration curve in Fig. 14, the required change of the notches' length will be $\Delta l_{1,2} \simeq\{0.7,3\} \mathrm{mm}$ and accordingly the scaling factors $K_{1,2}=\Delta \mathrm{AID}_{1,2, \text { min }} / \Delta l_{1,2}=$ $\{2.8,1\} \mathrm{dB} / \mathrm{mm}$. Finally, the angles of the dual-tapered selector are estimated from (6) as $\alpha_{1,2}=\left\{70^{\circ}, 45^{\circ}\right\}$. The resulting fabricated selector is visible in Fig. 16 (inset).

The measured calibration curve for $\delta \leftrightarrow \Delta$ AID (Fig. 16) is mostly linear and is in reasonable agreement with the expected $(S=1 \mathrm{~dB} / \mathrm{mm}$ )-slope line. In particular the measured AID appears slightly less sensitive in the first displacement interval $\left(S_{1} \approx 0.75 \mathrm{~dB} / \mathrm{mm}\right.$ for $0<\delta<2 \mathrm{~mm}$ ), while it is exactly sensitive as desired in the second part of displacement $\left(S_{2} \approx\right.$ $1 \mathrm{~dB} / \mathrm{mm}$, for $2<\delta<5 \mathrm{~mm}$ ).

\section{CONCLUSION}

Converting a spatial deformation into a change of a slot's or notch's form factor proved to be an effective mechanism for the wireless passive sensing of displacements with a resolution of a small fraction of millimeter. Assuming a typical commercial low-cost reader with $\Delta P_{\min }=0.5 \mathrm{~dB}$ power resolution, the sensor permits in principle to appreciate one-dimensional deformations as small as $\Delta d_{\min }(\xi)=\Delta P_{\min } / \Delta \xi=$ $\{55 \mu \mathrm{m}, 100 \mu \mathrm{m}\}$ in case the measurement indicator is the realized gain or the AID, respectively. Five-times smaller values $(11 \div 20 \mu \mathrm{m})$ could be even achieved with more expensive readers having $0.1 \mathrm{~dB}$ power sensitivities.

The proposed design methodology permits to fully control the range and the resolution of the sensor and can be in principle adapted to different kinds of slot's form factor as well as to different patch's shape. The described device is moreover suitable to be integrated with traditional visual crack-measurement equipments including a graduated stick, thus enabling an integrated observation. In particular, the size of the prototype is identical to that of a commercial crack-meter.

A true applicability of the device to real environments requires however additional research concerning the immunity of the radio-sensor to ambient agents that could induce uncertainty, such as humidity and dirtiness. Moreover, since in some cases the sensor should could be embedded in concrete near the surface, the degradation of performance in term of read range 
and sensitivity due to the concrete losses, will have to be properly addressed. Finally, future evolution of the device will consider ways to achieve sensing to two-dimensional deformations, for instance by employing a couplet of selectors and dual-chips tags.

\section{REFERENCES}

[1] N. Komninos, Intelligent Cities. London, U.K.: Spon Press London, 2003.

[2] L. Atzori, A. Iera, and G. Morabito, "The internet of things: A survey," Computer Networks, vol. 54, no. 15, pp. 2787-2805, 2010.

[3] P. C. Chang, A. Flatau, and S. C. Liu, "Review paper: Health monitoring of civil infrastructure," Structural Health Monitoring, vol. 2, no. 3, pp. 257-267, 2003.

[4] L. J. K. Lynch JP, "A summary review of wireless sensors and sensor networks for structural health monitoring," in Shock Vibr. Dig., Mar. 2006, vol. 38, pp. 91-128.

[5] A. Deivasigamani, A. Daliri, H. Chun, C. H. Wang, and J. John, "A review of passive wireless sensors for structural health monitoring," Modern Appl. Sci., vol. 7, no. 2, pp. 57-76, 2013.

[6] A. Daliri, A. Galehdar, S. John, C. H. Wang, W. S. Rowe, and K. Ghorbani, "Wireless strain measurement using circular microstrip patch antennas," Sensors Actuators A: Phys., vol. 184, no. 0, pp. 86-92, 2012.

[7] O. O. Rakibet, J. C. Batchelor, and S. W. Kelly, "Passive stretchable rfid tag," in Proc. Antennas Propag. Conf. (LAPC), Loughborough, U.K., Nov. 2012, pp. 1-4.

[8] S. Mita and A. Takahira, "A smart sensor using a mechanical memory for structural health monitoring of a damage-controlled building," Smart Mater. Structures, vol. 12, no. 2, pp. 204-209, 2003.

[9] A. Costanzo, D. Masotti, N. Arbizzani, V. Rizzoli, and F. Mastri, "A microwave sensor system based on reverse modeling of the array factor," in Proc. 6th European Conf. Antennas Propag. (EUCAP), Mar. 2012, pp. 3446-3449.

[10] M. Saafi, "Wireless and embedded carbon nanotube networks for damage detection in concrete structures," Nanotechnol., vol. 20, no. 39, p. 395502, 2009.

[11] K. M. Z. Shams and M. Ali, "Wireless power transmission to a buried sensor in concrete," IEEE Sensors J., vol. 7, pp. 1573-1577, 2007.

[12] O. Jonah and S. V. Georgakopoulos, "Wireless power transfer in concrete via strongly coupled magnetic resonance," IEEE Trans. Antennas Propag., vol. 61, no. 3, pp. 1378-1384, Mar. 2013.

[13] C. Occhiuzzi, C. Paggi, and G. Marrocco, "Passive RFID strain-sensor based on meander-line antennas," IEEE Trans. Antennas Propag., vol. 59, pp. 4836-4840, Dec. 2011

[14] S. Merilampi, P. Ruuskanen, T. Bjorninen, L. Ukkonen, and L. Sydanheimo, "Printed passive UHF RFID tags as wearable strain sensors," in Proc. 3rd Int. Symp. Appl. Sci. Biomed. Commun. Technol. (ISABEL), Nov. 2010, pp. 1-5.

[15] R. Bhattacharyya, C. Floerkemeier, and S. Sarma, "Towards tag antenna based sensing-an rfid displacement sensor," in Proc. IEEE Int. Conf. RFID, Apr. 2009, pp. 95-102.

[16] X. Yi, C. Cho, C.-H. Fang, J. Cooper, V. Lakafosis, R. Vyas, Y. Wang, R. T. Leon, and M. M. Tentzeris, "Wireless strain and crack sensing using a folded patch antenna," in Proc. 6th Euro. Conf. Antennas Propag. (EUCAP), Mar. 2012, pp. 1678-1681.
[17] S. S. P. Kalansuriya, R. Bhattacharyya, and N. Karmakar, "Towards chip-less rfid-based sensing for pervasive surface crack detection," in Proc. IEEE Int. Conf. RFID-Technol. Appl. (RFID-TA), 2012, pp. 46-51.

[18] S. Caizzone and G. Marrocco, "RFID-grids for deformation sensing," in Proc. IEEE Int. Conf. RFID (RFID), Apr. 2012, pp. 130-134.

[19] C. Occhiuzzi, S. Caizzone, and G. Marrocco, "Wireless sensing of things by passive RFID technology," IEEE Antennas Propagat. Mag., to be published.

[20] G. Marrocco, "RFID grids: Part i: Electromagnetic theory," IEEE Trans. Antennas Propag., vol. 59, pp. 1019-1026, Mar. 2011.

[21] S. Caizzone and G. Marrocco, "RFID grids: Part ii: Experimentations," IEEE Trans. Antennas Propag., vol. 59, pp. 2896-2904, Aug. 2011.

[22] S. Manzari, S. Pettinari, and G. Marrocco, "Miniaturised wearable uhf-rfid tag with tuning capability," Electron. Lett., vol. 48, no. 21, pp. $1325-1326,2012$.

[23] C. Occhiuzzi, S. Cippitelli, and G. Marrocco, "Modeling, design and experimentation of wearable RFID sensor tag," IEEE Trans. Antennas Propag., vol. 58, pp. 2490-2498, Aug. 2010.

[24] H. Rajagopalan and Y. Rahmat-Samii, "Conformal RFID antenna design suitable for human monitoring and metallic platforms," in Proc. 4th Euro. Conf, Antennas Propag. (EuCAP), Apr. 2010, pp. 1-5.

[25] R. Serrano, S. Capdevila, A. Aguasca, J. Romeu, and L. Jofre, "Multiport multiband coupling minimization for miniature antennas," in Proc. 3rd Euro. Conf. Antennas Propag. (EuCAP), Mar. 2009, pp. $1598-1602$.

[26] S. Manzari, A. Catini, C. Di Natale, and G. Marrocco, "Ambient sensing by chemical-loaded UHF-RFIDs," presented at the Euro Conf. Antennas Propag. (EUCAP), Sweden, 2013.

[27] M. Soutsos, J. Bungey, S. Millard, M. Shaw, and A. Patterson, "Dielectric properties of concrete and their influence on radar testing," NDT E Int., vol. 34, no. 6, pp. 419-425, 2001.

[28] C. Occhiuzzi and G. Marrocco, "Constrained-design of passive uhf rfid sensor antennas," IEEE Trans. Antennas Propag., vol. 61, pp. 2972-2980, Jun. 2013.

[29] C. Occhiuzzi, G. Contri, and G. Marrocco, "Design of implanted rfid tags for passive sensing of human body: The STENTag," IEEE Trans. Antennas Propag., vol. 60, pp. 3146-3154, Jul. 2012.

[30] G. Marrocco and F. Amato, "Self-sensing passive rfid: From theory to tag design and experimentation," in Proc. Euro. Microwave Conf. (EuMC), 2009, pp. 001-004.

C. Paggi, photograph and biography not available at the time of publication.

C. Occhiuzzi, photograph and biography not available at the time of publication.

G. Marrocco, photograph and biography not available at the time of publication. 